\title{
Gauge theories on a $2+2$ anisotropic lattice
}

\author{
Giuseppe Burgio, ${ }^{1}$ Alessandra Feo, ${ }^{1,2}$ Mike Peardon, ${ }^{1}$ and Sinéad M. Ryan ${ }^{1}$ \\ (TrinLat Collaboration) \\ ${ }^{1}$ School of Mathematics, Trinity College, Dublin 2, Ireland \\ ${ }^{2}$ Dipartimento di Fisica, Università di Parma and INFN Gruppo Collegato di Parma, \\ Parco Area delle Scienze, 7/A, 43100 Parma, Italy
}

(Received 19 March 2003; published 4 June 2003)

\begin{abstract}
The implementation of gauge theories on a four-dimensional anisotropic lattice with two distinct lattice spacings is discussed, with special attention to the case where two axes are finely and two axes are coarsely discretized. Feynman rules for the Wilson gauge action are derived and the renormalizability of the theory and the recovery of the continuum limit are analyzed. The calculation of the gluon propagator and the restoration of Lorentz invariance in on-shell states is presented to one-loop order in lattice perturbation theory for $S U\left(N_{c}\right)$ on both $2+2$ and $3+1$ lattices.
\end{abstract}

DOI: 10.1103/PhysRevD.67.114502

PACS number(s): 11.15.Ha

\section{INTRODUCTION}

The anisotropic lattice is a popular tool in numerical simulations. The usual approach is to make the temporal lattice spacing fine while keeping the spatial directions relatively coarse. The extra temporal resolution in this $3+1$ discretization scheme is useful when measuring two- and threepoint correlation functions of particles which decay rapidly. This is particularly useful for heavy hadrons and glueball states, for which the signal to noise ratio decreases rapidly with time. The glueball spectrum $[1,2]$ was an early success for this approach and, more recently, heavy quark systems have been studied using anisotropic lattices [3-14].

In this paper we consider a generalization of the anisotropic approach to include lattices with a $2+2$ discretization. The temporal and one spatial direction are made fine, keeping the remaining two spatial directions coarse. The motivation for this choice is to explore the feasibility of calculating decays which produce high-momentum daughter particles. These include the phenomenologically interesting transistions $B \rightarrow \pi l \nu$ and $B \rightarrow K^{*} \gamma$. Cabibbo-Kobayashi-Maskawa (CKM) matrix elements are determined from such exclusive decay processes through a combination of experimentally measured branching fractions and theoretical calculations of form factors. The light daughter hadrons in these decays have a nonzero momentum and so in a lattice calculation of the nonperturbative form factors there are cutoff effects proportional to this momentum in units of the lattice spacing. Therefore $B \rightarrow \pi l \nu$ has discretization errors proportional to $a p_{\pi}$ where $a$ is the lattice spacing. However, the range of momenta reliably reached by current experimental and lattice data do not overlap. Lattice calculations work best with $p_{\pi}$ $\leqslant 1 \mathrm{GeV}$ but the bulk of experimental data for the exclusive decay $B \rightarrow \pi l \nu$ lie at $p_{\pi} \geqslant 1.5 \mathrm{GeV}$. To avoid model dependence kinematic cuts can be applied, restricting the range of lattice data to momenta where the calculation is reliable [15], awaiting improved experimental results. Alternatively, the lattice data can be extrapolated to match experiment [16-18] but this introduces model dependence and increases substantially the systematic error in lattice calculations and therefore in $\left|V_{u b}\right|$. For $B \rightarrow K^{*} \gamma$ the situation is more acute since this decay happens at the maximum recoil momentum of the daughter meson, far from the range accessible to current lattice calculations. Therefore it is necessary to extrapolate well outside the range of reliable data $[19,20]$, once again introducing model dependence and increasing the systematic errors. For this reason it has not been widely studied using lattice methods and calculations to date have used isotropic lattices. In both cases the difficulty for lattice calculations is that errors proportional to the momentum of the order $a p$, grow quickly. These must be controlled to make phenomenologically relevant lattice calculations. The advantages then of the $2+2$ lattice are twofold. First, the fine temporal lattice spacing serves the same purpose as in the $3+1$ case: correlation functions should be accurately determined while keeping computational costs modest. Secondly, making one spatial direction fine and injecting all momentum along that direction keeps discretization errors of $\mathcal{O}(a p)$ small for high momenta.

The transition $B \rightarrow \pi l \nu$ has been calculated using a $3+1$ discretization scheme and the improved resolution in the temporal direction led to higher-momentum $\left(0 \leqslant p_{\pi}\right.$ $\leqslant 1.5 \mathrm{GeV}$ ) particles being reliably simulated [21]. Both this calculation and the isotropic calculations which were reviewed in Ref. [22] find that one of the largest systematic errors in the range of accessible momenta is due to the chiral extrapolation. The $2+2$ discretization does not address this issue but it is hoped that it will further extend the range of momentum available to lattice calculations and that statistical precision will be enhanced.

The paper is organized as follows. In Sec. II a general anisotropic formulation for $S U\left(N_{c}\right)$ Yang-Mills theory in four dimensions is presented. The differences between $3+1$ and $2+2$ discretisations are discussed in terms of the lattice symmetries and the parameter tuning required. Section III contains the framework of the perturbative calculation, already outlined in Ref. [23]. Although the goal is a $2+2$ discretization, the Feynman rules and the analytic procedure given are completely general and allow an exact treatment of the calculations. In Sec. IV we present our results and in- 
clude a comparison with other work. The challenging algebraic manipulations were performed on a computer using a symbolic code which handles the dependence on the anisotropy analytically. The core of the code is similar to that used in Ref. [24], while the treatment of the lattice integrals follows from Ref. [25]. Section V contains our conclusions, while some technical details are given in Appendixes A and $\mathrm{B}$.

\section{YANG-MILLS THEORIES ON A 2+2 ANISOTROPIC LATTICE}

In this section, formulations of $S U\left(N_{c}\right)$ Yang-Mills theory on general orthogonal lattice types are considered. A generalization of the Wilson action for $S U\left(N_{c}\right)$ Yang-Mills theory on the lattice is given by

$$
S_{\mathrm{W}}=\beta \sum_{n, \mu \nu} c_{\mu \nu}\left(1-\frac{1}{2 N_{c}} \operatorname{Tr}\left(P_{\mu \nu}(x)+P_{\mu \nu}^{\dagger}(x)\right)\right),
$$

where $\beta=2 N_{c} / g^{2}$ with $g^{2}$, the lattice coupling constant and $N_{c}$ the number of colors. $P_{\mu \nu}$ is the plaquette in the $(\mu, \nu)$ plane;

$$
P_{\mu \nu}(x)=U_{\mu}(x) U_{\nu}(x+\hat{\mu}) U_{\mu}^{\dagger}(x+\hat{\nu}) U_{\nu}^{\dagger}(x) .
$$

The six coefficients $c_{\mu \nu}$ in Eq. (1) will describe the anisotropy class.

If some sub-sets of these parameters are given identical values, the lattice action may have symmetries under the interchange of axes. In particular, if the two identities

$$
\begin{aligned}
& c_{12}=c_{23}=c_{31}\left(=c_{c c}\right) \\
& c_{41}=c_{42}=c_{43}\left(=c_{c f}\right)
\end{aligned}
$$

are imposed, the lattice action is symmetric under the cubic point group, and the $3+1$ anisotropy class is realized. Similarly, if the identity

$$
c_{13}=c_{14}=c_{23}=c_{24}\left(=c_{c f}\right)
$$

is imposed, the lattice action transforms trivially under elements of the group $C_{4 \nu} \otimes C_{4 \nu}$, with the first constituent group comprising the rotations and reflections in the $(1,2)$ plane and the second being those in the $(3,4)$ plane. This anisotropy class is denoted $2+2$.

For phenomenologically motivated reasons, as discussed in Sec. I, consideration will be restricted to these cases where only two distinct lattice spacings are permitted. The coarse and fine lattice spacing will be denoted $a_{c}$ and $a_{f}$ respectively. In this paper the overall scale $a$ is chosen to be $a_{f}$. The implementation of a $3+1$ anisotropy, in which the lattice spacing in the temporal direction is made fine keeping the spatial lattice spacing coarse, has been widely discussed in the literature [26,27].

In the continuum, the corresponding sub-groups of $O(4)$, the rotations in Euclidean space that are generated by demanding the same symmetries in the coefficients of the continuum dimension-four operators, are $O(3)$ for the class de- scribed in Eq. (3) and $O(2) \otimes O(2)$ for Eq. (4). If the operators which transform trivially under these symmetries are enumerated there is one operator for the Euclidean group, $\left(\operatorname{Tr} F_{\mu \nu} F_{\mu \nu}\right.$ ), two operators for the $O(3)$ spatial rotation group (most conveniently denoted $\operatorname{Tr} E_{i} E_{i}$ and $\operatorname{Tr} B_{i} B_{i}$ for chromoelectric and chromomagnetic) and three operators for the $O(2) \otimes O(2) \quad$ case $\quad\left(\operatorname{Tr} F_{f f^{\prime}} F_{f f^{\prime}}, \quad \operatorname{Tr} F_{f c} F_{f c} \quad\right.$ and $\left.\operatorname{Tr} F_{c c^{\prime}} F_{c c^{\prime}}\right)$.

The recovery of Euclidean invariance in the low-energy physics of an anisotropic lattice requires a parameter tuning, in contrast to the isotropic case. Additional differences arise between the $3+1$ lattices, for which a one-parameter tuning suffices and $2+2$ lattices which necessitate a two-parameter tuning. The importance of parameter tuning, in particular for the $2+2$ case is emphasized in Sec. II A.

Finally, it is interesting to note that taking the anisotropy $\xi \rightarrow \infty$ yields, up to a gauge transformation, the Hamiltonian limit of the theory on a $3+1$ lattice. For the $2+2$ anisotropy class, this limit cannot be taken naively; a theory in which only two of the four dimensions are discretized is not regularized.

\section{A. 2+2: the need for tuning}

As the continuum limit is approached for the $3+1$ lattice theory, the physics of the Euclidean invariant Yang-Mills theory is reproduced, provided care is taken to account for the different grid spacings, $a_{c}$ and $a$. This can be achieved by tuning the relative weights of the two sets of coefficients in Eq. (3) to ensure the ratio of scales, $\xi=a_{c} / a$ measured by a physical probe takes its desired value or alternatively, an arbitrary choice of the two parameters $c_{c c}$ and $c_{c f}$ can be taken and the ratio of scales measured post hoc. The $3+1$ anisotropic theory is certain to be in the same universality class as the desired theory since the single free parameter (the relative weight of $c_{c c}$ and $c_{c f}$ ) determines the ratio of scales $\xi$.

For the $2+2$ case, an important distinction arises; while there are only two distinct dimension-four continuum operators in the $3+1$ class, there are three for the $2+2$ case. As a result, the recovery of a Euclidean invariant continuum theory is not guaranteed since there are two free parameters and only a single ratio of scales. As a result, the general $2+2$ lattice theories can lie in a larger universality class than the continuum four-dimensional Yang-Mills theory.

For this reason, care must be taken to ensure the recovery of Euclidean invariance in the continuum limit for a $2+2$ simulation. The relative weights of the three operators in the action must be determined to ensure Lorentz invariance in on-shell Green's functions. This tuning can be achieved perturbatively or by restoring symmetries in a nonperturbative calculation of, for example the static inter-quark potential. In the remainder of this paper, perturbation theory is used to determine the parameters in the action. A paper describing the nonperturbative tuning of the parameters is in preparation [28].

\section{B. $c_{\mu \nu}$ from perturbation theory}

At the tree level, it is straightforward to determine the values of the coefficients in the action. For the $3+1$ lattice 
action, they are $c_{c c}=1 / \xi, c_{c f}=\xi$ and for the $2+2$ case, they are $c_{c c}=1 / \xi^{2}, c_{c f}=1$ and $c_{f f}=\xi^{2}$. The one-loop definitions of $c_{\mu \nu}$ can then be parametrized as

$$
c_{\mu \nu}^{(2+2)}=\left\{\begin{array}{lll}
\xi^{2} & \left(1+\eta_{f f}^{(1)} g^{2}+O\left(g^{4}\right)\right), & \mu, \nu \in \mathrm{f}, \\
1 & \left(1+\eta_{c f}^{(1)} g^{2}+O\left(g^{4}\right)\right), & \mu \in \mathrm{c}(\mathrm{f}), \nu \in \mathrm{f}(\mathrm{c}), \\
\frac{1}{\xi^{2}} & \left(1+\eta_{c c}^{(1)} g^{2}+O\left(g^{4}\right)\right), & \mu, \nu \in \mathrm{c},
\end{array}\right.
$$

and

$$
c_{\mu \nu}^{(3+1)}=\left\{\begin{array}{lll}
\xi & \left(1+\eta_{c f}^{(1)} g^{2}+O\left(g^{4}\right)\right), & \mu \in \mathrm{c}(\mathrm{f}), \nu \in \mathrm{f}(\mathrm{c}), \\
\frac{1}{\xi} & \left(1+\eta_{c c}^{(1)} g^{2}+O\left(g^{4}\right)\right), & \mu, \nu \in \mathrm{c} .
\end{array}\right.
$$

In both cases, an overall multiplicative weight can be absorbed into a redefinition of the field integration variables, and this is redundant in the action-tuning procedure since a symmetry is being enforced in on-shell Green's functions. This change of variables will become important once matrixelement matching of gluon fields is being performed.

\section{PERTURBATION THEORY}

We can now proceed to discuss Feynman rules for a general four-dimensional anisotropic Wilson-like action, already outlined in Ref. [23]. We follow the notation given in Ref. [29] and the interpretation of the anisotropy as a difference in momentum cutoffs will be our guideline. The matching of the lattice gluon action in Eq. (1) with its continuum counterpart is made clearer by expressing the link variables $U_{\mu}(n)=e^{i \phi_{\mu}(n)}$ in terms of dimensionless fields

$$
\phi_{\mu}(n)=\phi_{\mu}^{b}(n) T^{b},
$$

where $T^{b}$ are the $S U\left(N_{c}\right)$ generators in the fundamental representation satisfying the relations $\left[T^{a}, T^{b}\right]=i f^{a b c} T^{c}$ and $\operatorname{Tr}\left(T^{a} T^{b}\right)=\frac{1}{2} \delta^{a b}$. The dimensionful gluon fields $A_{\mu}(x)$ can then be reintroduced in Eq. (7) using the relation

$$
\phi_{\mu}^{b}(n)=g a \xi_{\mu} A_{\mu}^{b}(x)
$$

where

$$
\xi_{\mu}= \begin{cases}1, & \mu \in \mathrm{f} \\ \xi, & \mu \in \mathrm{c}\end{cases}
$$

is the anisotropy index. By taking into account the Jacobian

$$
\sum_{n}=\frac{1}{\xi^{d} a^{4}} \sum_{x}
$$

where $d$ denotes the number of coarse directions, one easily obtains Eqs. (5) and (6). The continuum limit of the Eq. (1) is then written as

$$
\begin{aligned}
S_{\mathrm{W}} & =\sum_{n} \frac{1}{4} c_{\mu \nu} \hat{F}_{\mu \nu}^{b}(n) \hat{F}_{\mu \nu}^{b}(n)+\mathcal{O} \\
& =\frac{1}{4} \sum_{x} F_{\mu \nu}^{b}(x) F_{\mu \nu}^{b}(x)+O\left(a^{2}\right),
\end{aligned}
$$

where $\mathcal{O}$ is an irrelevant operator in the continuum involving terms with three or more gluon fields from which the nontrivial contribution to the Feynman rules will arise, while

$$
\hat{F}_{\mu \nu}^{b}(n)=\hat{\partial}_{\mu}^{R} \phi_{\nu}^{b}(n)-\hat{\partial}_{\nu}^{R} \phi_{\mu}^{b}(n)-g f^{b c d} \phi_{\mu}^{c}(n) \phi_{\nu}^{d}(n),
$$

and

$$
\hat{\partial}_{\mu}^{R} \varphi(n)=\varphi(n+\hat{\mu})-\varphi(n)
$$

are the dimensionless field strength and lattice right derivative, whereas

$$
F_{\mu \nu}^{b}(x)=\partial_{\mu}^{R} A_{\nu}^{b}(x)-\partial_{\nu}^{R} A_{\mu}^{b}(x)-g f^{b c d} A_{\mu}^{c}(x) A_{\nu}^{d}(x),
$$

and

$$
\partial_{\mu}^{R} \varphi(x)=\frac{1}{a \xi_{\mu}}\left(\varphi\left(x+a \xi_{\mu} \hat{\mu}\right)-\varphi(x)\right)
$$

are their dimensionful equivalents.

As is well known, the Wilson action has $O\left(a^{2}\right)$ discretization errors in the evaluation of various physical quantities. In addition, the lattice regularization gives rise to finite renormalization coefficients when compared to other continuum schemes. These effects can be reduced by adding irrelevant operators in the action which reduce discretization artifacts, or by improving the convergence of renormalization coefficients to better match continuum quantities $[30,31]$. In any of these cases an exact and completely algebraic treatment of the Feynman rules is always viable, even at orders higher than one [32-36]. As for the lattice integrals, while a completely numerical evaluation presents no problem at first order, any one-plaquette action will change the structure of the $O\left(a^{2}\right)$ (irrelevant) terms in the four and higher gluon vertices but will not change the propagators, the three-gluon vertex, the measure or the gauge-fixing terms, which are all fixed by the Haar measure and the naive matching with the continuum limit [37]. This means that once the first-order analytic technique for the Wilson action is developed it can be applied to any other one-plaquette action. For gauge actions with Wilson loops which extend two grid spacings, the technique given in Ref. [25] (for fermion propagators) can be adapted while for actions with even larger loops, suitable techniques can be developed in the same spirit. Using coordinate-space methods, analytic results are also available for higher orders $[32,38,39]$. Finally, mean-link improvement schemes [40] are straightforward to implement once the corresponding Feynman diagram is written down. 


\section{A. The gauge fixing and the gluon propagator}

For perturbation theory it is necessary to choose a gauge fixing and proceed to define the other parts of the action needed to write the Feynman rules. In analogy with the isotropic case we choose the gauge-fixing term such that

$$
S_{g f}=\frac{1}{\alpha} \sum_{x} \operatorname{Tr}\left(\partial_{\mu}^{L} A_{\mu}\right)^{2},
$$

where $\quad \partial_{\mu}^{L} \varphi(x)=1 /\left(a \xi_{\mu}\right)\left(\varphi(x)-\varphi\left(x-a \xi_{\mu} \hat{\mu}\right)\right)$. It is straightforward to show that

$$
S_{g f}=\frac{1}{\tilde{\alpha}} \sum_{n} \operatorname{Tr} \mathcal{F}^{2} \text { for } \mathcal{F}=b_{\mu}^{(l)} \hat{\partial}_{\mu}^{L} \phi_{\mu}(n),
$$

where now $\hat{\partial}_{\mu}^{L} \varphi(n)=\varphi(n)-\varphi(n-\hat{\mu})$ and

$$
b_{\mu}^{(l)}= \begin{cases}\xi^{l+1}, & \mu \in \mathrm{f}, \\ \xi^{l-1}, & \mu \in \mathrm{c},\end{cases}
$$

gives the desired form once $\alpha=\xi^{-2 l+d-2} \tilde{\alpha}$. The choice $l$ $=(d-2) / 2$ would be easiest but we shall see in the following it is not the most convenient. The gluon propagator can now be calculated from the two field terms in Eq. (12) and the definition of the gauge fixing in Eq. (17). The functional form is similar to the isotropic case; namely,

$$
\begin{gathered}
\mu, a \text { eleleee } \nu, b \\
=\frac{\delta^{a b}}{\hat{k}^{2}}\left(\delta_{\mu \nu}-(1-\alpha) \frac{\hat{k}_{\mu} \hat{k}_{\nu}}{\hat{k}^{2}}\right),
\end{gathered}
$$

where

$$
\begin{aligned}
& \hat{k}_{\mu}=\frac{2}{a \xi_{\mu}} \sin \left(\frac{a \xi_{\mu}}{2} k_{\mu}\right), \\
& \hat{k}^{2}=\sum_{\mu} \hat{k}_{\mu}^{2}, \\
& k_{\mu} \in\left(-\frac{\pi}{a \xi_{\mu}}, \frac{\pi}{a \xi_{\mu}}\right),
\end{aligned}
$$

are the dimensionful lattice momenta spanning the anisotropic Brillouin zones. All the anisotropy is now encapsulated in this form of the Brillouin zone. This will prove to be a constant pattern: for any vertex or propagator which has a continuum analogue, the form in terms of the (anisotropic) dimensionful momenta will resemble the isotropic ones, since the asymmetry now lies solely in the different momenta cutoffs.

\section{B. The Haar measure}

On the lattice the functional integral is obtained by integrating over the dimensionless gauge links, but to do perturbation theory it must be expressed in terms of the dimension- ful gauge fields. The Jacobian resulting from the expression of the Haar measure in terms of Eq. (7) is [29]

$$
\begin{gathered}
J=\sqrt{\operatorname{det}\left(\frac{1}{2} M M^{\dagger}\right)}, \\
M\left(\widetilde{\phi}_{\mu}(n)\right)=\frac{1-e^{-i \widetilde{\phi}_{\mu}(n)}}{i \widetilde{\phi}_{\mu}(n)}, \\
\widetilde{\phi}_{\mu}(n)=\phi_{\mu}^{a}(n) t^{a},
\end{gathered}
$$

where the $\phi_{\mu}^{a}(n)$ are the same as in Eq. (7) and the $t^{a}$ are the adjoint generators of $S U\left(N_{c}\right)$. Reexpressing $J$ as $e^{-S_{\text {meas }}}$ gives

$$
S_{\text {meas }}=-\frac{1}{2} \sum_{n, \mu} \operatorname{Tr} \log \left(\frac{2\left(1-\cos \widetilde{\phi}_{\mu}(n)\right)}{\widetilde{\phi}_{\mu}^{2}(n)}\right) .
$$

When expanded to lowest order in the fields (which is all that is needed for one-loop two-point function calculations) this reads

$$
S_{\text {meas }} \simeq \frac{1}{4 !} \sum_{n, \mu} N_{c} \delta^{a b} \phi_{\mu}^{a}(n) \phi_{\mu}^{b}(n),
$$

which putting back the dimensionful fields gives the vertex

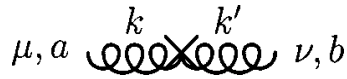

$$
\begin{aligned}
& =-(2 \pi)^{4} \delta^{4}\left(k+k^{\prime}\right) \frac{g^{2}}{a^{2}} \frac{N_{c}}{12} \delta_{\mu \nu} \delta^{a b} \frac{\xi_{\mu}^{2}}{\xi^{d}} .
\end{aligned}
$$

By convention all gluon momenta are incoming.

\section{Fadeev-Popov ghost fields}

The Faddeev-Popov determinant, which forces the integration only on a section of the gauge orbits, must also be included in the action. Using $\mathcal{F}$ in Eq. (17) to enforce the gauge condition on $\phi_{\mu}$ and using the response of the gauge fields on the lattice to (infinitesimal) gauge transformations [29]

$$
\hat{D}_{\mu}(\phi)=M^{-1}\left(\widetilde{\phi}_{\mu}\right) \hat{\partial}_{\mu}^{R}+i \widetilde{\phi}_{\mu}
$$

where $\widetilde{\phi}_{\mu}$ and the inverse adjoint-valued operator, $M$ are given in Eq. (21), we obtain

$$
S_{\mathrm{gh}}=-\sum_{n, \mu} \overline{\hat{c}}^{a}(n) b_{\mu}^{(l)} \hat{\partial}_{\mu}^{L} \hat{D}_{\mu}^{a b}(\phi) \hat{c}^{b}(n),
$$

where $\hat{c}$ and $\overline{\hat{c}}$ are the dimensionless lattice ghost fields, introduced to make Eq. (26) local. From the expansion of $M^{-1}$,

$$
M^{-1}\left(\widetilde{\phi}_{\mu}\right)=\mathbb{I}_{\mathrm{adj}}+\frac{i}{2} \widetilde{\phi}_{\mu}-\frac{1}{12} \widetilde{\phi}_{\mu}^{2}+\cdots
$$


and by reintroducing dimensions, Eq. (26) can be written as

$$
\begin{aligned}
S_{\mathrm{gh}} \simeq & -\xi^{l-1} \sum_{x} \bar{c}^{a}(x) \delta^{a b} \partial_{\mu}^{L} \partial_{\mu}^{R} c^{b}(x)+g f^{a b c} \bar{c}^{a}(x) \partial_{\mu}^{L} \\
& \times\left[A_{\mu}^{c}(x)\left(1+\frac{a \xi_{\mu}}{2} \partial_{\mu}^{R}\right)\right] c^{b}(x) \\
& +\frac{1}{2 !} \frac{g^{2} a^{2} \xi_{\mu}^{2}}{12} \delta_{\mu \nu}\left\{t^{c}, t^{d}\right\}_{a b} \partial_{\mu}^{R} \bar{c}^{a}(x) \partial_{\mu}^{R} c^{b}(x) A_{\mu}^{c}(x) A_{\nu}^{d}(x),
\end{aligned}
$$

by including only terms which are relevant for one-loop twopoint functions. The factor $\xi^{l-1}$ at the front of $S_{\mathrm{gh}}$ gives rise to spurious coefficients with no continuum analogue which however, cancel in any graph with no ghost outer legs. Alternatively the coefficients can be absorbed in a redefinition of the ghost fields $\xi^{(l-1) / 2} c \rightarrow c$ or by setting $l=1$, thus rescaling the lattice gauge-fixing parameter $\tilde{\alpha}=\xi^{4-d} \alpha$. For each of these alternatives the ghost propagator and the two ghost one- and two-gluon vertices are

$$
\begin{aligned}
& a \cdots \cdots \\
& =\delta^{a b} \frac{1}{\hat{k}^{2}}, \\
& p_{p, a}^{p^{\prime}, b}{ }_{\text {eQQ }}^{k} \mu, c \\
& =i g(2 \pi)^{4} \delta^{4}\left(k+p-p^{\prime}\right) f^{a b c} \hat{p}_{\mu}^{\prime} \tilde{p}_{\mu}, \\
& { }_{k, \mu, c}^{p, b}{ }_{\Phi^{\prime}, \nu, d}^{\rho_{e}, a} \\
& =\frac{1}{12} g^{2} a^{2}(2 \pi)^{4} \delta^{4}\left(k+k^{\prime}+p-p^{\prime}\right)\left\{t^{c}, t^{d}\right\}_{a b} \delta_{\mu \nu} \xi_{\mu}^{2} \hat{p}_{\mu} \hat{p}_{\nu}^{\prime},
\end{aligned}
$$

with $\tilde{p}_{\mu}=\cos \frac{1}{2} p_{\mu} a \xi_{\mu}$. As usual the vertex which has a continuum analogue carries no explicit dependence on the anisotropy.

\section{Gluon vertices}

Taking into account the three-gluon terms arising from Eq. (1), one of which comes from the irrelevant operator $\mathcal{O}$ defined in Eq. (11) gives
$S_{\mathrm{W}}^{(3)}=g \sum_{x} f^{a b c} \delta_{\lambda \nu}\left(A_{\mu}^{a}(x)+\frac{a \xi_{\mu}}{2} \partial_{\nu}^{R} A_{\mu}^{a}(x)\right) \partial_{\mu}^{R} A_{\nu}^{b}(x) A_{\lambda}^{c}(x)$

which, after a Fourier transformation, leads to an expression equivalent to the isotropic case

$$
\begin{aligned}
& k, \mu, a \\
& k^{\prime}, \nu, b \\
& =i g(2 \pi)^{4} \delta^{4}\left(k+k^{\prime}+k^{\prime \prime}\right) f^{a b c}\left(\delta_{\nu \lambda} \widehat{\left(k^{\prime \prime}-k^{\prime}\right.}\right)_{\mu} \widetilde{k}_{\nu} \\
& \left.+\delta_{\mu \lambda} \widehat{\left(k-k^{\prime \prime}\right)}{ }_{\nu} \widetilde{k^{\prime}}{ }_{\lambda}+\delta_{\mu \nu} \widehat{\left(k^{\prime}-k\right)}{ }_{\lambda} \widetilde{k^{\prime \prime}}{ }_{\mu}\right) \text {. }
\end{aligned}
$$

Taking the four-gluon terms arising from Eq. (1), mostly coming from $\mathcal{O}$ (see Ref. [29] for details), and rechecking the cancellations which must occur due to Bose symmetry, one finds the four-gluon vertex whose expression is given in Appendix A.

\section{E. One-loop correction vertex}

The one-loop corrections to the action coming from Eqs. (5) and (6) give rise to an extra vertex which reads

$$
\begin{aligned}
& \mu, a \stackrel{k}{k^{\prime}} \stackrel{k^{\prime}}{\nu, b} \\
& =-(2 \pi)^{4} \delta^{4}\left(k+k^{\prime}\right) g^{2} \delta^{a b}\left(\delta_{\mu \nu} \sum_{\rho} \eta_{\mu \rho}^{(1)} \hat{k}_{\rho}^{2}-\eta_{\mu \nu}^{(1)} \hat{k}_{\mu} \hat{k}_{\nu}\right),
\end{aligned}
$$

where

$$
\eta_{\mu \nu}^{(1)}= \begin{cases}\eta_{f f}^{(1)}, & \mu, \nu \in \mathrm{f}, \\ \eta_{c f}^{(1)}, & \mu \in \mathrm{c}(\mathrm{f}), \nu \in \mathrm{f}(\mathrm{c}), \\ \eta_{c c}^{(1)}, & \mu, \nu \in \mathrm{c} .\end{cases}
$$

In the $3+1$ case one can either set $\eta_{f f}^{(1)}$ to zero or leave it free, as by the traceless property of the vertex it will always cancel.

\section{F. The continuum limit and anisotropic renormalization}

The calculation of the one-loop corrections to the gluon self-energy, $\Sigma_{\mu \nu, a b}(p)$, using the Wilson action involves five Feynman diagrams [41] and Eq. (34). Each diagram is a function of the external momenta $p$ and can be written as

$$
G(p)=\int \frac{d^{4} k}{(2 \pi)^{4}} F(k, p)
$$

where $k$ is the integration momenta. Since we are interested in the continuum limit of Eq. (36), if the integral is ultravio- 
let convergent we can simply substitute the function $F(k, p)$ with its continuum equivalent. Otherwise, if Eq. (36) is divergent and contains only massive propagators so that $F(k, p)$ is finite for any set of momenta going to zero, one can use the lattice version of the BPHZ technique [42-44] by writing

$$
\begin{aligned}
G(p)= & \int \frac{d^{4} k}{(2 \pi)^{4}}\left[F(k, p)-\sum_{n=0}^{n_{F}} \frac{1}{n !} p_{\mu_{1}} \ldots p_{\mu_{n}} \frac{\partial}{\partial p_{\mu_{1}}}\right. \\
& \left.\times\left.\cdots \frac{\partial}{\partial p_{\mu_{n}}} F(k, p)_{p=0}\right|_{p=0}\right] \\
& +\int \frac{d^{4} k}{(2 \pi)^{4}} \sum_{n=0}^{n_{F}} \frac{1}{n !} p_{\mu_{1}} \ldots p_{\mu_{n}} \frac{\partial}{\partial p_{\mu_{1}}} \\
& \times\left.\ldots \frac{\partial}{\partial p_{\mu_{n}}} F(k, p)\right|_{p=0} \equiv G^{c}(p)+G^{L}(p),
\end{aligned}
$$

where $n_{F}$ is the degree of divergence of the diagram. The first term in Eq. (37) is ultraviolet finite $[43,44]$ and therefore its continuum limit can be taken, making it independent of $\xi$. All the effects of the lattice regularization remain in the second term, which is simply a polynomial in the external momenta with coefficients given by zero-momentum lattice integrals.

On the other hand, if a diagram contains massless propagators, as in our case, more care is needed: indeed an expansion around $p=0$ can give rise to infrared divergences. A simple recipe is to introduce an intermediate infrared regularization. Given an anisotropic cutoff the introduction of a mass, $m$ in the propagators is the most suitable solution $[25,32,45]$, while dimensional regularization is popular in the literature for the isotropic case $[33,41] . G^{c}(p)$ and $G^{L}(p)$ are then divergent for $m \rightarrow 0$ separately but the divergences cancel in the sum.

\section{RESULTS}

In this section we present the results of the one-loop correction to the gluon propagator using the Wilson action in the Feynman gauge for a general anisotropic lattice in four dimensions. Applying the procedure explained in Sec. IV A we calculate the values of the coefficients which restore on-shell Lorentz invariance, where the definition and treatment of the lattice integrals $\mathcal{B}_{\xi}$ is given in Appendix B.

\section{A. On-shell Lorentz invariance}

As explained in Sec. II, the one-loop propagator obtained from the Feynman rules given in Sec. III will not in general satisfy Lorentz invariance. The free parameters in Eq. (35) must be tuned to restore the symmetry $[26,27,46]$. From Eq.
(34) it is clear that not every Lorentz-breaking term can be cancelled, as expressions of the form $\delta_{\mu \nu} / a^{2}$ and $\delta_{\mu \nu} p_{\mu}^{2}$ arise from each diagram. The first nontrivial result of our calculation is that, for any value of $d$ and $\xi$, such terms cancel exactly when the diagrams are summed up, just as in the isotropic case, independently of the tuning procedure.

The remaining Lorentz-breaking artifacts arising from the mixing of longitudinal and transverse field components have the correct structure and can be cured by tuning. We choose to fix the parameters in the action by demanding the recovery of Lorentz invariance for on-shell physical soft gluon modes, imposing that the two physical eigenvalues of the one-loop propagator vanish for $E^{2}=p^{2}$. Since this is a gauge-invariant condition we restrict ourselves to a particular gauge. We have chosen the Feynman gauge for which $\alpha=1$. By injecting the gluon momentum in any possible direction and calculating the eigenvalues and eigenvectors of the propagator we obtain a general condition for $\eta_{c c}^{(1)}-\eta_{c f}^{(1)}$ and $\eta_{f f}^{(1)}$ $-\eta_{c f}^{(1)}$, independent of the direction of $p_{\mu}$. The value of $\eta_{c f}^{(1)}$ remains unconstrained as it can be reabsorbed in a oneloop $\beta$ shift.

To identify the physical eigenmodes, the momenta directions with residual symmetry are first identified. In the case of the $2+2$ lattice, this corresponds to any momenta in the coarse-coarse or fine-fine planes. Then for more general momenta, the axis of propagation was smoothly varied away from these symmetric cases, and the eigenmodes continuously traced. This investigation lead to a generalization of the polarization condition, $p_{\mu} \epsilon_{\mu}=0$ for on-shell gluon polarization vector $\epsilon_{\mu}$. The resulting $2+2$ lattice polarization condition is $p_{\mu} Z_{\mu \nu} \epsilon_{\nu}=0$, with $Z_{\mu \nu}$ is the diagonal matrix $Z_{\mu \nu}=\delta_{\mu \nu}\left(Z_{c} \delta_{\mu c}+Z_{f} \delta_{\mu f}\right)$ and $Z_{c}, Z_{f}$ the coarse and fine gluon field renormalization coefficients. $\delta_{\mu f}$ and $\delta_{\mu c}$ are one (zero) if $\mu$ is fine (coarse).

\section{B. The one-loop coefficients for the restoration of Lorentz invariance}

For $2+2$ anisotropic lattice the one-loop coefficients for the restoration of Lorentz invariance are

$$
\begin{aligned}
\eta_{c c}^{(1)}-\eta_{c f}^{(1)}= & -\frac{1}{2 N_{c}}\left[\mathcal{B}_{\xi}^{c}(1,1)-\frac{1}{4}\right] \\
& +N_{c}\left[-\frac{1}{16}+\frac{\mathcal{B}_{\xi}(1)}{6}\left(\frac{7}{2}+\frac{1}{\xi^{2}}\right)\right. \\
& +\frac{\mathcal{B}_{\xi}^{c}(1,1)}{4}-\frac{\mathcal{B}_{\xi}^{c}(2,1)}{3}\left(2+\frac{5}{2 \xi^{4}}+\frac{11}{2 \xi^{2}}\right) \\
& \left.+\frac{\mathcal{B}_{\xi}^{f}(2,1,1)}{6}\left(\frac{1}{2}+\xi^{2}\right)\right],
\end{aligned}
$$

and 
TABLE I. $N_{c}$-polynomial coefficients of $\eta_{c c}^{(1)}-\eta_{c f}^{(1)}$ and $\eta_{f f}^{(1)}-\eta_{c f}^{(1)}$ for $\xi=1, \ldots, 10$ on a $2+2$ anisotropic lattice.

\begin{tabular}{lcccc}
\hline \hline$\xi$ & \multicolumn{2}{c}{$\eta_{c c}^{(1)}-\eta_{c f}^{(1)}$} & \multicolumn{2}{c}{$\eta_{f f}^{(1)}-\eta_{c f}^{(1)}$} \\
\hline $1 / N_{c}$ & 0 & $N_{c}$ & $1 / N_{c}$ & $N_{c}$ \\
\hline & -0.1161042701 & 0.0958601237 & 0 & 0 \\
3 & -0.1886258210 & 0.1529850132 & 0.0775690169 & -0.0741548219 \\
4 & -0.2391230676 & 0.1920838712 & 0.1010941459 & -0.1044390683 \\
5 & -0.2774799637 & 0.2215115752 & 0.1107973557 & -0.1217185361 \\
6 & -0.3083219799 & 0.2450412380 & 0.1156439679 & -0.1335092263 \\
7 & -0.3340937184 & 0.2646297423 & 0.1183899089 & -0.1424129673 \\
8 & -0.3562244833 & 0.2814071688 & 0.1200891686 & -0.1495669092 \\
9 & -0.3756169823 & 0.2960809268 & 0.1212112364 & -0.1555537098 \\
10 & -0.3928761275 & 0.3091218866 & 0.1219898822 & -0.1607076435 \\
\hline \hline
\end{tabular}

$$
\begin{aligned}
\eta_{f f}^{(1)}-\eta_{c f}^{(1)}= & \frac{1}{2 N_{c}}\left[\frac{1}{4}-\frac{1}{2 \xi^{2}}+\frac{\mathcal{B}_{\xi}^{c}(1,1)}{\xi^{4}}\right]-\frac{N_{c}}{2}\left[\frac{1}{4}\left(\frac{1}{2}-\frac{1}{\xi^{2}}\right)\right. \\
& +\mathcal{B}_{\xi}(1)\left(\frac{1}{2}+\frac{1}{3 \xi^{2}}\right)+\frac{\mathcal{B}_{\xi}^{c}(1,1)}{2 \xi^{4}} \\
& \left.-\mathcal{B}_{\xi}^{c}(2,1)\left(\frac{5}{3 \xi^{4}}+\frac{1}{\xi^{2}}+\frac{1}{6}\right)-\frac{\mathcal{B}_{\xi}^{f}(2,1,1)}{3 \xi^{2}}\right],
\end{aligned}
$$

which diverge logarithmically with $\xi$, while for $3+1$ we have

$$
\begin{aligned}
\eta_{c c}^{(1)}-\eta_{c f}^{(1)}= & \frac{N_{c}}{\xi}\left[\frac{\mathcal{B}_{\xi}(1)}{6}\left(\frac{\xi^{2}}{3}+\frac{19}{6}+\frac{7}{2 \xi^{2}}\right)\right. \\
& +\frac{\mathcal{B}_{\xi}^{c}(1,1)}{4}\left(1+\frac{1}{\xi^{2}}\right)-\mathcal{B}_{\xi}^{c}(2,1)\left(\frac{1}{3}+\frac{11}{6 \xi^{2}}\right. \\
& \left.\left.+\frac{5}{2 \xi^{4}}\right)-\frac{1}{8}\right]-\frac{1}{2 \xi N_{c}}\left[\mathcal{B}_{\xi}^{c}(1,1)\left(1+\frac{1}{\xi^{2}}\right)-\frac{1}{2}\right],
\end{aligned}
$$

which agrees with the general result of Ref. [27] and the $N_{c}=3$ result of Ref. [46]. The techniques used by both groups involve extrapolations in some suitable parameter, absent in our treatment. The functions $\mathcal{B}_{\xi}$ are defined in Appendix B. Using the results given there the Hamiltonian limit $\xi \rightarrow \infty$ can be treated analytically and agrees again with Ref. [27]. The difference in the parameters, $\eta_{c c}^{(1)}-\eta_{c f}^{(1)}$ and $\eta_{f f}^{(1)}$ $-\eta_{c f}^{(1)}$ appears as a polynomial in $N_{c}$ with terms proportional to $1 / N_{c}$ and $N_{c}$ only, as expected. Numerical values for the coefficients in this polynomial for a range of anisotropies are given to ten decimal places in Tables I and II.

\section{The gluon self-energy in the Feynman gauge}

Once the values in Eqs. (38), (39) and (40) are inserted, the one-loop correction to the gluon self-energy reads

$$
\begin{aligned}
\Sigma_{\mu \nu, a b}^{(1)}(p)= & g^{2} \delta^{a b}\left(\delta_{\mu \nu} p^{2}-p_{\mu} p_{\nu}\right) \\
& \times\left\{A-\eta_{c f}^{(1)}-B\left(\delta_{\mu c} \delta_{\nu c}-\delta_{\mu f} \delta_{\nu f}\right)\right\} .
\end{aligned}
$$

For $2+2$ we have

$$
\begin{aligned}
A= & -\frac{1}{8 N_{c}}+N_{c}\left[\frac{1}{16}+\frac{\mathcal{B}_{\xi}(1)}{2}\left(\frac{1}{3}-\frac{1}{4 \xi^{2}}\right)\right. \\
& \left.+\frac{\mathcal{B}_{\xi}^{c}(2,1)}{4 \xi^{2}}\left(\frac{7}{3 \xi^{2}}-1\right)-\frac{\mathcal{B}_{\xi}^{f}(2,1,1)}{12}+\frac{5}{3} X(\xi)\right] \\
B & =N_{c}\left[\frac{\mathcal{B}_{\xi}(1)}{12}\left(1+\frac{1}{2 \xi^{2}}\right)-\frac{\mathcal{B}_{\xi}^{c}(2,1)}{4 \xi^{2}}\left(1+\frac{1}{\xi^{2}}\right)\right],
\end{aligned}
$$

while for $3+1$

TABLE II. $N_{c}$-polynomial coefficients of $\eta_{c c}^{(1)}-\eta_{c f}^{(1)}$ for $\xi$ $=1, \ldots, 10$ on a $3+1$ anisotropic lattice.

\begin{tabular}{lcc}
\hline \hline$\xi$ & \multicolumn{2}{c}{$\eta_{c c}^{(1)}-\eta_{c f}^{(1)}$} \\
\hline $1 / N_{c}$ & $N_{c}$ \\
\hline 1 & 0 & 0 \\
3 & -0.0853420430 & 0.0663515978 \\
4 & -0.1202379609 & 0.0892976526 \\
5 & -0.1389282597 & 0.1007025039 \\
6 & -0.1504977336 & 0.1074686585 \\
7 & -0.1583451793 & 0.1119335310 \\
8 & -0.1640119208 & 0.1150960459 \\
9 & -0.1682939409 & 0.1174518583 \\
10 & -0.1716426764 & 0.1192740369 \\
\hline \hline
\end{tabular}


TABLE III. $N_{c}$-polynomial coefficients of $\eta_{c c}^{(1)}-\eta_{c f}^{(1)}$ and $\eta_{f f}^{(1)}-\eta_{c f}^{(1)}$ for $\xi=1, \ldots, 10$ on a $2+2$ anisotropic lattice using mean (coarse) plaquette improvement.

\begin{tabular}{lcccc}
\hline \hline$\xi$ & \multicolumn{2}{c}{$\eta_{c c}^{(1)}-\eta_{c f}^{(1)}$} & \multicolumn{2}{c}{$\eta_{f f}^{(1)}-\eta_{c f}^{(1)}$} \\
\hline $1 / N_{c}$ & $-1 / 16$ & $N_{c}$ & $1 / N_{c}$ & $N_{c}$ \\
\hline 2 & -0.2366564052 & 0.2164122588 & $1 / 16$ & $-1 / 16$ \\
3 & -0.3454387314 & 0.3097979237 & 0.1981211519 & -0.1947069547 \\
4 & -0.4211846015 & 0.3741454050 & 0.2579070564 & -0.2612519788 \\
5 & -0.4787199456 & 0.4227515571 & 0.2928588896 & -0.3037800700 \\
6 & -0.5249829699 & 0.4617022280 & 0.3168839498 & -0.3347492082 \\
7 & -0.5636405777 & 0.4941766016 & 0.3350508989 & -0.3590739573 \\
8 & -0.5968367250 & 0.5220194105 & 0.3496360278 & -0.3791137684 \\
9 & -0.6259254734 & 0.5463894180 & 0.3618234781 & -0.3961659514 \\
10 & -0.6518141912 & 0.5680599503 & 0.3722983733 & -0.4110161346 \\
\hline \hline
\end{tabular}

$$
\begin{aligned}
A= & \frac{1}{2 N_{c} \xi}\left[\frac{\mathcal{B}_{\xi}^{c}(1,1)}{\xi^{2}}-\frac{1}{2}\right]+\frac{N_{c}}{\xi}\left[\frac{1}{8}+\frac{5 \xi}{3} X(\xi)-\frac{\mathcal{B}_{\xi}^{c}(1,1)}{4 \xi^{2}}\right. \\
& \left.+\frac{\mathcal{B}_{\xi}^{c}(2,1)}{4 \xi^{2}}\left(\frac{7}{\xi^{2}}+\frac{1}{3}\right)+\frac{\mathcal{B}_{\xi}(1)}{6}\left(\frac{1}{3}-\frac{5}{2 \xi^{2}}\right)\right] \\
B & =\frac{N_{c}}{\xi}\left[\frac{\mathcal{B}_{\xi}(1)}{6}\left(\frac{1}{2}+\frac{1}{\xi^{2}}\right)-\frac{\mathcal{B}_{\xi}^{c}(2,1)}{4 \xi^{2}}\left(1+\frac{3}{\xi^{2}}\right)\right]
\end{aligned}
$$

where

$$
X(\xi)=\frac{1}{16 \pi^{2}}\left(-\log \left(a^{2} p^{2}\right)+F_{0}(\xi)-\gamma_{E}+\frac{28}{9}\right) .
$$

quantities such as the $\Lambda$ parameter or matrix elements. After including the difference, the physical modes will still be transverse.

Setting $\xi=1$ we recover from Eq. (41) the well-known value of the gluon self-energy $[32,33,41]$

$$
\begin{aligned}
\Sigma_{\mu \nu, a b}(p)= & \delta^{a b}\left(\delta_{\mu \nu} p^{2}-p_{\mu} p_{\nu}\right) \\
& \times\left[1-g^{2}\left\{\frac{N_{c}}{16 \pi^{2}}\left(-\frac{5}{3} \log \left(p^{2} a^{2}\right)+\frac{28}{9}\right)\right.\right. \\
& \left.\left.-\frac{1}{8 N_{c}}+N_{c}\left(\frac{7}{72} Z_{0}+\frac{5}{48 \pi^{2}}\left(F_{0}-\gamma_{E}\right)+\frac{1}{16}\right)\right\}\right] \\
& +O\left(g^{4}\right),
\end{aligned}
$$

We stress that the term proportional to $B$ in Eq. (41) does not break Lorentz invariance but arises from the difference in the renormalizations of the fine and coarse fields $A_{\mu}$. This difference must be taken into account when calculating physical

where $Z_{0}$ and $F_{0}$ are standard integrals in perturbation theory on the isotropic lattice (see e.g. Ref. [45]). In order to calculate coefficients at the one-loop level in mean-link improved

TABLE IV. $N_{c}$-polynomial coefficients of $\eta_{c c}^{(1)}-\eta_{c f}^{(1)}$ and $\eta_{f f}^{(1)}-\eta_{c f}^{(1)}$ for $\xi=1, \ldots, 10$ on a $2+2$ anisotropic lattice using mean (coarse) link improvement. $Z_{0}$ is defined in Sec. IV B.

\begin{tabular}{lcccc}
\hline \hline$\xi$ & \multicolumn{2}{c}{$\eta_{c c}^{(1)}-\eta_{c f}^{(1)}$} & \multicolumn{2}{c}{$\eta_{f f}^{(1)}-\eta_{c f}^{(1)}$} \\
\hline $1 / N_{c}$ & $N_{c}$ & $1 / N_{c}$ & $N_{c}$ \\
\hline 1 & $-3 Z_{0} / 8$ & $3 Z_{0} / 8$ & $3 Z_{0} / 8$ & $-3 Z_{0} / 8$ \\
2 & -0.2304051667 & 0.2101610203 & 0.1918699134 & -0.1884557162 \\
3 & -0.3391678963 & 0.3035270885 & 0.2516362213 & -0.2549811436 \\
4 & -0.4150758989 & 0.3680367024 & 0.2867501870 & -0.2976713674 \\
5 & -0.4727526305 & 0.4167842420 & 0.3109166347 & -0.3287818930 \\
6 & -0.5191216331 & 0.4558408912 & 0.3291895621 & -0.3532126205 \\
7 & -0.5578576084 & 0.4883936322 & 0.3438530585 & -0.3733307991 \\
8 & -0.5911125215 & 0.5162952070 & 0.3560992746 & -0.3904417479 \\
9 & -0.6202462174 & 0.5407101620 & 0.3666191173 & -0.4053368786 \\
10 & -0.6461700016 & 0.5624157607 & 0.3758456617 & -0.4185310183 \\
\hline \hline
\end{tabular}


TABLE V. $N_{c}$-polynomial coefficients of $\eta_{c c}^{(1)}-\eta_{c f}^{(1)}$ for $\xi$ $=1, \ldots, 10$ on a $3+1$ anisotropic lattice using mean (coarse) plaquette improvement.

\begin{tabular}{lcc}
\hline \hline$\xi$ & \multicolumn{2}{c}{$\eta_{c c}^{(1)}-\eta_{c f}^{(1)}$} \\
\hline $1 / N_{c}$ & $N_{c}$ \\
\hline 1 & $-1 / 16$ & $1 / 16$ \\
2 & -0.1694788603 & 0.1504884150 \\
3 & -0.2118450434 & 0.1809047350 \\
4 & -0.2337180290 & 0.1954922732 \\
5 & -0.2468908748 & 0.2038617997 \\
6 & -0.2556482395 & 0.2092365912 \\
7 & -0.2618777619 & 0.2129618870 \\
8 & -0.2665309579 & 0.2156888753 \\
9 & -0.2701369251 & 0.2177682856 \\
10 & -0.2730125217 & 0.2194047833 \\
\hline \hline
\end{tabular}

perturbation theory, the evaluation of either the plaquette, $\left\langle\operatorname{Tr} P_{\mu \nu}\right\rangle$ or the link trace in Landau gauge, $\left\langle\operatorname{Tr} U_{\mu}\right\rangle$ is required. We find

$$
\begin{aligned}
\frac{1}{N_{c}}\left\langle\operatorname{Tr} P_{c c}\right\rangle= & 1-\frac{g^{2}}{2 \xi^{d-2}}\left(N_{c}-\frac{1}{N_{c}}\right) \mathcal{B}_{\xi}^{c}(1,1), \\
\frac{1}{N_{c}}\left\langle\operatorname{Tr} P_{f c}\right\rangle= & 1-\frac{g^{2}}{4 \xi^{d}}\left(N_{c}-\frac{1}{N_{c}}\right)\left(\xi^{2} \mathcal{B}_{\xi}^{f}(1,1)\right. \\
& \left.+\mathcal{B}_{\xi}^{c}(1,1)\right), \\
\frac{1}{N_{c}}\left\langle\operatorname{Tr} P_{f f}\right\rangle= & 1-\frac{g^{2}}{2 \xi^{d}}\left(N_{c}-\frac{1}{N_{c}}\right) \mathcal{B}_{\xi}^{f}(1,1),
\end{aligned}
$$

and

$$
\begin{aligned}
\frac{1}{N_{c}}\left\langle\operatorname{Tr} U_{c}\right\rangle= & 1-\frac{g^{2}}{4 \xi^{d}}\left(N_{c}-\frac{1}{N_{c}}\right) \\
& \times\left[\xi^{2} \mathcal{B}_{\xi}(1)-\mathcal{B}_{\xi}^{c}(2,1)\left(1-\frac{1}{\alpha}\right)\right], \\
\frac{1}{N_{c}}\left\langle\operatorname{Tr} U_{f}\right\rangle= & 1-\frac{g^{2}}{4 \xi^{d}}\left(N_{c}-\frac{1}{N_{c}}\right) \\
& \times\left[\mathcal{B}_{\xi}(1)-\mathcal{B}_{\xi}^{f}(2,1)\left(1-\frac{1}{\alpha}\right)\right],
\end{aligned}
$$

where $\mathcal{B}_{\xi}^{f}(q, 1)$ satisfies Eq. (B4). Numerical values of the $N_{c}$-polynomial coefficients, $\eta_{c c}^{(1)}, \eta_{c f}^{(1)}$ and $\eta_{f f}^{(1)}$ for a range of anisotropies and including these improvement terms are given in Tables III, IV, V, and VI.
TABLE VI. $N_{c}$-polynomial coefficients of $\eta_{c c}^{(1)}-\eta_{c f}^{(1)}$ for $\xi$ $=1, \ldots, 10$ on a $3+1$ anisotropic lattice using mean (coarse) link improvement. $Z_{0}$ is defined in Sec. IV B.

\begin{tabular}{lcc}
\hline \hline$\xi$ & \multicolumn{2}{c}{$\eta_{c c}^{(1)}-\eta_{c f}^{(1)}$} \\
\hline $1 / N_{c}$ & $N_{c}$ \\
\hline 1 & $-3 Z_{0} / 8$ & $3 Z_{0} / 8$ \\
2 & -0.1643550433 & 0.1453645980 \\
3 & -0.2068380749 & 0.1758977666 \\
4 & -0.2288323401 & 0.1906065843 \\
5 & -0.2420838939 & 0.1990548187 \\
6 & -0.2508913882 & 0.2044797400 \\
7 & -0.2571539394 & 0.2082380645 \\
8 & -0.2618297768 & 0.2109876942 \\
9 & -0.2654518384 & 0.2130831989 \\
10 & -0.2683392407 & 0.2147315023 \\
\hline \hline
\end{tabular}

\section{CONCLUSIONS}

In this paper, a generalization of the Wilson discretization to an anisotropic lattice with two coarse and two fine directions has been described. In particular, an important distinction between the $2+2$ anisotropy and the well-established $3+1$ case has been emphasized. The difference is that the coefficients in the $2+2$ action must be determined before simulation to ensure Lorentz invariance, while any $3+1$ action leads to a Lorentz-invariant theory once the ratio of scales $\xi$ is determined. We are currently investigating nonperturbative techniques for computing these coefficients in the $2+2$ case [28].

The main result of the paper was to compute these coefficients to first order in perturbation theory. While the focus of the calculation was on determining the Feynman rules for the $2+2$ lattice Wilson gauge action, a more general prescription was developed to allow the $3+1$ case to be investigated as well. This allowed us to check our results against previously published work. The results in a mean-link improvement scheme were presented.

The usefulness of this scheme arises from the need to make accurate calculations of form factors and matrix elements at high momentum. This paper establishes the tools for perturbation theory calculations on the $2+2$ anisotropic lattice, which will be important later when computing the matching factors to link calculations of weak-decay matrix elements to their continuum counterparts. As part of this program, quark fields on $2+2$ anisotropic lattices are under consideration.

\section{ACKNOWLEDGMENTS}

This work was funded by Enterprise-Ireland grants $\mathrm{SC} /$ 2001/306 and SC/2001/307. We would like to thank H. Panagopoulos for helpful discussions. 


$$
\begin{aligned}
& \text { APPENDIX A: THE FOUR GLUON VERTEX } \\
& q, \nu, b \quad r, \lambda, c \\
& k, \mu, a \stackrel{s, \rho, d}{ } \\
& =-g^{2}\left\{\sum _ { e } f ^ { a b e } f ^ { c d e } \left\{\delta_{\mu \lambda} \delta_{\nu \rho}\left[\widetilde{(q-s)} \widetilde{(k-r)}_{\nu}-\frac{a^{4}}{12} \xi_{\mu}^{2} \xi_{\nu}^{2} \hat{k}_{\nu} \hat{q}_{\mu} \hat{r}_{\nu} \hat{s}_{\mu}\right]-\delta_{\mu \rho} \delta_{\nu \lambda}\left[\widetilde{(q-r)} \widetilde{\mu}_{\mu}(k-s)_{\nu}-\frac{a^{4}}{12} \xi_{\mu}^{2} \xi_{\nu}^{2} \hat{k}_{\nu} \hat{q}_{\mu} \hat{r}_{\mu} \hat{s}_{\nu}\right]\right.\right. \\
& +\frac{a^{2}}{6} \delta_{\nu \lambda} \delta_{\nu \rho} \xi_{\nu}^{2} \widehat{(s-r)} \hat{k}_{\nu} \tilde{q}_{\mu}-\frac{a^{2}}{6} \delta_{\mu \lambda} \delta_{\mu \rho} \xi_{\mu}^{2} \widehat{(s-r)} \hat{q}_{\mu} \widetilde{k}_{\nu}+\frac{a^{2}}{6} \delta_{\mu \nu} \delta_{\mu \rho} \xi_{\rho}^{2} \widehat{(q-k)} \hat{r}_{\rho} \tilde{s}_{\lambda}-\frac{a^{2}}{6} \delta_{\mu \nu} \delta_{\mu \lambda} \xi_{\lambda}^{2} \widehat{(q-k)} \hat{s}_{\lambda} \tilde{r}_{\rho} \\
& \left.\left.+\frac{a^{2}}{12} \delta_{\mu \nu} \delta_{\mu \lambda} \delta_{\mu \rho} \xi_{\mu}^{2} \sum_{\sigma} \widehat{(q-k)}_{\sigma}^{(s-r)} \sigma\right\}+(b \leftrightarrow c, \nu \leftrightarrow \lambda, q \leftrightarrow r)+(b \leftrightarrow d, \nu \leftrightarrow \rho, q \leftrightarrow s)\right\}+\frac{g^{2}}{12} a^{4}\left\{\frac { 2 } { N _ { c } } \left(\delta^{a b} \delta^{c d}+\delta^{a c} \delta^{b d}\right.\right. \\
& \left.\left.+\delta^{a d} \delta^{b c}\right)+\sum_{e}\left(d^{a b e} d^{c d e}+d^{a c e} d^{b d e}+d^{a d e} d^{b c e}\right)\right\}\left\{\delta_{\mu \nu} \delta_{\mu \lambda} \delta_{\mu \rho} \xi_{\mu}^{2} \sum_{\sigma} \xi_{\sigma}^{2} \hat{k}_{\sigma} \hat{q}_{\sigma} \hat{r}_{\sigma} \hat{s}_{\sigma}-\delta_{\mu \nu} \delta_{\mu \lambda} \xi_{\mu}^{2} \xi_{\rho}^{2} \hat{k}_{\rho} \hat{q}_{\rho} \hat{r}_{\rho} \hat{s}_{\mu}\right. \\
& -\delta_{\mu \nu} \delta_{\mu \rho} \xi_{\mu}^{2} \xi_{\lambda}^{2} \hat{k}_{\lambda} \hat{q}_{\lambda} \hat{s}_{\lambda} \hat{r}_{\mu}-\delta_{\mu \lambda} \delta_{\mu \rho} \xi_{\mu}^{2} \xi_{\nu}^{2} \hat{k}_{\nu} \hat{r}_{\nu} \hat{s}_{\nu} \hat{q}_{\mu}-\delta_{\nu \lambda} \delta_{\nu \rho} \xi_{\mu}^{2} \xi_{\nu}^{2} \hat{q}_{\mu} \hat{r}_{\mu} \hat{s}_{\mu} \hat{k}_{\nu}+\delta_{\mu \nu} \delta_{\lambda \rho} \xi_{\mu}^{2} \xi_{\lambda}^{2} \hat{k}_{\lambda} \hat{q}_{\lambda} \hat{r}_{\mu} \hat{s}_{\mu} \\
& \left.+\delta_{\mu \lambda} \delta_{\nu \rho} \xi_{\mu}^{2} \xi_{\nu}^{2} \hat{k}_{\nu} \hat{r}_{\nu} \hat{q}_{\mu} \hat{s}_{\mu}+\delta_{\mu \rho} \delta_{\nu \lambda} \xi_{\mu}^{2} \xi_{\nu}^{2} \hat{k}_{\nu} \hat{s}_{\nu} \hat{q}_{\mu} \hat{r}_{\mu}\right\} \text {. }
\end{aligned}
$$

\section{APPENDIX B: BOSONIC INTEGRAL EVALUATION}

Apart from the denominators in Eq. (19) and (29), all the $\xi$ dependence in the evaluation of Feynman diagrams is polynomial in $\xi$ and $1 / \xi$. Having changed integration variables to the asymmetric Brillouin zone, only lattice zeromomentum integrals of the form

$$
\mathcal{B}_{\xi}\left(q, n_{1}, n_{2}, n_{3}, n_{4}\right)=\int_{-\pi}^{\pi} \frac{d^{4} k}{(2 \pi)^{4}} \frac{\hat{k}_{x}^{2 n_{1}} \hat{k}_{y}^{2 n_{2}} \hat{k}_{z}^{2 n_{3}} \hat{k}_{t}^{2 n_{4}}}{D_{B}(k, m)^{q}}
$$

remain. In Eq. (B1), $q$ and $n_{i}$ are positive integers, $\hat{k}_{\mu}$ $=2 \sin \left(k_{\mu} / 2\right)$ and the $\xi$ dependence is in the inverse bosonic propagator

$$
D_{B}(k, m)=\sum_{\mu \in f} \hat{k}_{\mu}^{2}+\frac{1}{\xi^{2}} \sum_{\mu \in c} \hat{k}_{\mu}^{2}+m^{2} .
$$

alone. To evaluate these integrals, we adapt a technique given in Refs. [25], [45]. In the following, when one of the $n_{i}$ is zero it is omitted as an argument of $\mathcal{B}_{\xi}$, while, where no confusion can arise, the index in $n_{\mu}$ is dropped and $\mathcal{B}_{\xi}^{f}, \mathcal{B}_{\xi}^{c}$ or $\mathcal{B}_{\xi}^{f c}$ denotes integrals whose numerators have a fine, coarse or mixed momentum. In Refs. [25], [45], a set of recursion relations was defined to reduce every relevant integral to a linear combination of three basic integrals. Although similar relations exist in the asymmetric case, the lack of a complete symmetry among the indices, $n_{i}$ makes this reduction more difficult. The relations can still be used to reduce the number of integrals which must be calculated considerably and to prove the cancellations of Lorentzbreaking terms. A first set can be obtained by expanding the trivial identity

$$
\mathcal{B}_{\xi}\left(q-n, n_{a}, \ldots, n_{b}\right)=\int_{-\pi}^{\pi} \frac{d^{4} k}{(2 \pi)^{4}} \frac{\hat{k}_{a}^{2 n_{a}} \ldots \hat{k}_{b}^{2 n_{b}} D_{B}(k, m)^{n}}{D_{B}(k, m)^{q}},
$$

which gives relations of the type

$$
(4-d) \mathcal{B}_{\xi}^{f}(q, 1)+\frac{d}{\xi^{2}} \mathcal{B}_{\xi}^{c}(q, 1)=\mathcal{B}_{\xi}(q-1)-m^{2} \mathcal{B}_{\xi}(q),
$$

$$
\begin{aligned}
& (4-d) \mathcal{B}_{\xi}^{f}(q, 2)+(4-d)(3-d) \mathcal{B}_{\xi}^{f}(q, 1,1) \\
& +\frac{2 d(4-d)}{\xi^{2}} \mathcal{B}_{\xi}^{f c}(q, 1,1)+\frac{d}{\xi^{4}} \mathcal{B}_{\xi}^{c}(q, 2) \\
& +\frac{d(d-1)}{\xi^{4}} \mathcal{B}_{\xi}^{c}(q, 1,1)=\mathcal{B}_{\xi}(q-2)-2 m^{2} \mathcal{B}_{\xi}(q-1) \\
& +m^{4} \mathcal{B}_{\xi}(q)
\end{aligned}
$$

and so on. Furthermore, when $r>1$, using the identity 
TABLE VII. $\mathcal{B}_{\xi}(1)$ for $2+2$ and $3+1$ as a function of $\xi$ to $10^{-32}$ precision.

\begin{tabular}{lc}
\hline \hline$\xi$ & $2+2$ \\
\hline 1 & 0.15493339023106021408483720810745 \\
2 & 0.27309284159872576605248340464151 \\
3 & 0.34489265148380372504682107682350 \\
4 & 0.39476479072982726208009053119503 \\
5 & 0.43269826887073920594692758485023 \\
6 & 0.46325286965085016997908881189910 \\
7 & 0.48882308585244885903279712081054 \\
8 & 0.51080767194929084936132635888190 \\
9 & 0.53009088018361126653278475943989 \\
10 & 0.54726616387832592956739420406785 \\
\hline$\xi$ & $3+1$ \\
\hline 1 & 0.15493339023106021408483720810745 \\
2 & 0.39746855267384293273601515359921 \\
3 & 0.63909144650207181551150685543306 \\
4 & 0.87599824804148552213829648851546 \\
5 & 1.10989753717092264667311285543088 \\
6 & 1.34197554748773397230022959566745 \\
7 & 1.57290464634371239724254250061935 \\
8 & 1.80307278894183022754245621178021 \\
9 & 2.03271449270324135251760022607330 \\
10 & 2.26197832054321458404301146488387 \\
\hline \hline
\end{tabular}

$$
\begin{aligned}
\frac{\left(\hat{k}_{\mu}^{2}\right)^{r}}{D_{B}(k, m)^{q}}= & 4 \frac{\left(\hat{k}_{\mu}^{2}\right)^{r-1}}{D_{B}(k, m)^{q}}+2 \xi_{\mu}^{2} \frac{\left(\hat{k}_{\mu}^{2}\right)^{r-2}}{q-1} \\
& \times \sin k_{\mu} \frac{\partial}{\partial k_{\mu}} \frac{1}{D_{B}(k, m)^{q-1}},
\end{aligned}
$$

and integrating by parts, we obtain the relation

$$
\begin{aligned}
\mathcal{B}_{\xi}\left(q, \ldots, r_{\mu}, \ldots\right)= & \xi_{\mu}^{2} \frac{r-1}{q-1} \mathcal{B}_{\xi}\left(q-1, \ldots, r_{\mu}-1, \ldots\right) \\
& -\xi_{\mu}^{2} \frac{4 r-6}{q-1} \mathcal{B}_{\xi}\left(q-1, \ldots, r_{\mu}-2, \ldots\right) \\
& +4 \mathcal{B}_{\xi}\left(q, \ldots, r_{\mu}-1, \ldots\right), \quad \text { (B7) }
\end{aligned}
$$

which depends on the index $\mu=f, c$. A final set of relations is found by using the trivial fact that the numerator cannot have more than four different arguments, e.g.

$$
\begin{aligned}
\mathcal{B}_{\xi}(q, 1,1,1,1)= & (d-4) \mathcal{B}_{\xi}\left(q+1,2_{f}, 1,1,1\right) \\
& +\frac{d}{\xi^{2}} \mathcal{B}_{\xi}\left(q+1,1,1,1,2_{c}\right) \\
& +m^{2} \mathcal{B}_{\xi}(q+1,1,1,1,1) .
\end{aligned}
$$

The $2+2$ case has the bonus relation, $1 / \xi \mathcal{B}_{\xi}=\xi \mathcal{B} \frac{1}{\xi}$ which is relevant to the numerical evaluation of a range of integrals. A similar relation would map $3+1$ integrals to $1+3$. With the
TABLE VIII. $F_{0}(\xi)$ for $2+2$ and $3+1$ as a function of $\xi$ to $10^{-28}$ precision.

\begin{tabular}{lc}
\hline \hline$\xi$ & $2+2$ \\
\hline 1 & 4.3692252338747587180021767477 \\
2 & 3.1818862274504847285875097585 \\
3 & 2.2336373613788360098511237787 \\
4 & 1.5519382904759206782491885601 \\
5 & 1.0353940883292892812839278453 \\
6 & 0.6235592513202110800048370509 \\
7 & 0.2823597812415587497126918746 \\
8 & -0.0084789916018349072700020647 \\
9 & -0.2617758427992475365501484308 \\
10 & -0.4860763765593393395653061782 \\
\hline$\xi$ & $3+1$ \\
\hline 1 & 4.3692252338747587180021767478077 \\
2 & 3.0013807239614706751354255882375 \\
3 & 2.0763302182432342962706829473432 \\
4 & 1.4343462876751234663331108882378 \\
5 & 0.9504439561174006940006958674003 \\
6 & 0.5632462682606760634612041417967 \\
7 & 0.2405570583083765791881447111856 \\
8 & -0.036178339243301342993891362519 \\
9 & -0.278533198254716148526058151986 \\
10 & -0.494190516782956896533394268235 \\
\hline \hline
\end{tabular}

help of these relations we can reduce the propagator calculation to four converging integrals, $\mathcal{B}_{\xi}(1), \mathcal{B}_{\xi}^{c}(1,1), \mathcal{B}_{\xi}^{c}(2,1)$ and $\mathcal{B}_{\xi}^{f}(2,1,1)$, the last of which vanishes for $3+1$, and one infrared diverging integral, $\mathcal{B}_{\xi}(2)$.

For numerical calculation, setting $n=\sum_{i} n_{i}$ and using the well-known Schwinger representation [41], the integrals are rewritten as

$$
\begin{aligned}
\mathcal{B}_{\xi}\left(q, n_{1}, n_{2}, n_{3}, n_{4}\right)= & \frac{(-1)^{n}}{2^{q-n} \Gamma(q)} \int_{0}^{\infty} d \lambda \lambda^{q-1} e^{-m^{2} \lambda / 2} \\
& \times \prod_{\mu=1}^{4}\left[\frac{d^{n_{\mu}}}{d x^{n_{\mu}}} \exp ^{-x} I_{0}(x)\right]_{x=\lambda / d_{\mu}^{2}},
\end{aligned}
$$

with

$$
\frac{d^{n}}{d x^{n}} \exp ^{-x} I_{0}(x)=\frac{e^{-x}}{2^{n-1}} \sum_{k=0}^{n}(-1)^{n-k} M_{3}^{n, k} I_{k}(x),
$$

where $M_{3}^{n, k}$ are partition multinomial coefficients and $I_{k}(x)$ are modified Bessel functions of the first kind $[47,48]$. Since we want to focus attention on the massless case $\left(m^{2} \rightarrow 0\right)$ and keeping only the nonvanishing terms either divergent or finite, power-counting shows that the integrals, $\mathcal{B}_{\xi}\left(q, n_{1}, n_{2}, n_{3}, n_{4}\right)$ for $q-n \leqslant 1$, are infrared finite. As a result their value can be directly calculated from Eq. (B9) by 
setting $m^{2}=0$. Numerical evaluation to, say, 32 digits becomes trivial with the use of a numerical integration package available with programs like MATHEMATICA or MAPLE (see Table VII). Furthermore, simple dimensional arguments give $\mathcal{B}_{\xi}\left(q, n_{f}, n_{c}\right)=O\left((\xi \log \xi)^{\left.q-n_{f} / \xi\right)}\right.$ for $q>n_{f}$ and $\mathcal{B}_{\xi}\left(q, n_{f}, n_{c}\right)$ $=O\left(\xi^{q-n_{f}}\right)$ for $q \leqslant n_{f}$ for the $2+2$ case while for $3+1$, $\mathcal{B}_{\xi}\left(q, n_{f}, n_{c}\right)=O\left(\xi^{q-n_{f}}\right)$. From this, the asymptotic behavior of the one-loop corrections can be easily obtained, by noting $\lim _{\xi \rightarrow \infty} \mathcal{B}_{\xi}^{c}(2,1) /\left(\xi^{2} \mathcal{B}_{\xi}(1)\right)=1 / 6$ for $3+1$.

We can now describe the computation of the relevant part of $\mathcal{B}_{\xi}\left(q, n_{1}, n_{2}, n_{3}, n_{4}\right), q-n \geqslant 2$, which is infrared divergent. Using the asymptotic expansion for large $x$ of $I_{\nu}(x)$

$$
I_{\nu}(x) \approx \frac{e^{x}}{\sqrt{2 \pi x}} \sum_{k=0}^{\infty} \frac{(-1)^{k} \Gamma(\nu+k+1 / 2)}{(2 x)^{k} k ! \Gamma(\nu-k+1 / 2)},
$$

the leading and sub-leading behavior is easily determined. This can be reexpressed in terms of $\Gamma\left(i, m^{2}\right), i=0 \ldots q-n$ -2 whose integral representation can be directly subtracted from the integrand, leaving an $m^{2} \rightarrow 0$ converging integral. For example, in the case $n=0$, the constants $b_{i}$ are defined as the expansion of $I_{0}(x)^{4}$, which are rational numbers multiplied by $\pi^{-2}$. The divergent part of $\mathcal{B}_{\xi}(q)$ is then given by

$$
\frac{\xi^{d}}{\Gamma(q)} \sum_{i=2}^{q-1} \frac{b_{i-2} \Gamma(q-i)}{2^{i}\left(m^{2}\right)^{q-i}}-\frac{\xi^{d} b_{q-2}}{2^{p} \Gamma(q)} \log m^{2} .
$$

Following the literature, the finite contribution to

$$
\mathcal{B}_{\xi}(2)=\frac{\xi^{d}}{16 \pi^{2}}\left(-\log m^{2}-\gamma_{E}+F_{0}(\xi)\right)+O\left(m^{2}\right)
$$

is calculated first and the finite part of the other integrals is defined up to it. Using

$$
\Gamma\left(0, m^{2}\right)=-\log m^{2}-\gamma_{E}-\sum_{n=1}^{\infty} \frac{\left(-m^{2}\right)^{n}}{n n !}
$$

and taking the $\lim _{m^{2} \rightarrow 0}$ yields

$$
\begin{aligned}
F_{0}(\xi)= & \frac{4 \pi^{2}}{\xi^{d}} \int_{0}^{2} d \lambda \lambda \exp ^{-\left(4-d+d / \xi^{2}\right) \lambda} I_{0}^{4-d}(\lambda) I_{0}^{d}\left(\frac{\lambda}{\xi^{2}}\right) \\
& +\int_{2}^{\infty} d \lambda\left(\frac{4 \pi^{2}}{\xi^{d}} \lambda \exp ^{-\left(4-d+d / \xi^{2}\right) \lambda} I_{0}^{4-d}(\lambda)\right. \\
& \left.\times I_{0}^{d}\left(\frac{\lambda}{\xi^{2}}\right)-\frac{1}{\lambda}\right) .
\end{aligned}
$$

For example

$$
\begin{gathered}
\mathcal{B}_{\xi}(3)=\frac{\xi^{d}}{32 \pi^{2} m^{2}}+\frac{\xi^{d}}{128 \pi^{2}}\left(\log m^{2}+\gamma_{E}-F_{0}(\xi)\right)+f \mathcal{B}_{\xi}(3), \\
\mathcal{B}_{\xi}^{f}(3,1)=\frac{\xi^{d}}{64 \pi^{2}}\left(-\log m^{2}-\gamma_{E}+F_{0}(\xi)\right)+f \mathcal{B}_{\xi}^{f}(3,1) \\
\mathcal{B}_{\xi}^{c}(3,1)=\frac{\xi^{d+2}}{64 \pi^{2}}\left(-\log m^{2}-\gamma_{E}+F_{0}(\xi)\right)+f \mathcal{B}_{\xi}^{c}(3,1)
\end{gathered}
$$

which using Eq. (B5) and taking $\lim _{m^{2} \rightarrow 0} m^{2} \mathcal{B}_{\xi}(3)$, must satisfy

$$
(4-d) f \mathcal{B}_{\xi}^{f}(3,1)+\frac{d}{\xi^{2}} f \mathcal{B}_{\xi}^{c}(3,1)+\frac{\xi^{d}}{32 \pi^{2}}=0 .
$$

Numerical values for $\mathcal{B}_{\xi}(1)$ and $F_{0}$ are presented for a range of anisotropies in Tables VII and VIII.
[1] C.J. Morningstar and M.J. Peardon, Phys. Rev. D 60, 034509 (1999).

[2] C.J. Morningstar and M.J. Peardon, Phys. Rev. D 56, 4043 (1997).

[3] J. Fingberg, Phys. Lett. B 424, 343 (1998).

[4] X. Liao and T. Manke, Phys. Rev. D 65, 074508 (2002).

[5] K.J. Juge, J. Kuti, and C.J. Morningstar, Nucl. Phys. B (Proc. Suppl.) 63, 543 (1998).

[6] K.J. Juge, J. Kuti, and C.J. Morningstar, Phys. Rev. Lett. 82, 4400 (1999).

[7] K.J. Juge, J. Kuti, and C.J. Morningstar, Nucl. Phys. B (Proc. Suppl.) 83, 503 (2000).

[8] I.T. Drummond, R.R. Horgan, T. Manke, and H.P. Shanahan, Nucl. Phys. B (Proc. Suppl.) 73, 336 (1999).

[9] CP-PACS Collaboration, T. Manke et al., Phys. Rev. Lett. 82, 4396 (1999).
[10] CP-PACS Collaboration, T. Manke, Nucl. Phys. B (Proc. Suppl.) 86, 397 (2000).

[11] I.T. Drummond, N.A. Goodman, R.R. Horgan, H.P. Shanahan, and L.C. Storoni, Phys. Lett. B 478, 151 (2000).

[12] UKQCD Collaboration, S. Collins et al., Phys. Rev. D 64, 055002 (2001).

[13] J. Harada, A.S. Kronfeld, H. Matsufuru, N. Nakajima, and T. Onogi, Phys. Rev. D 64, 074501 (2001).

[14] S. Hashimoto and M. Okamoto, hep-lat/0302012.

[15] A.X. El-Khadra, A.S. Kronfeld, P.B. Mackenzie, S.M. Ryan, and J.N. Simone, Phys. Rev. D 64, 014502 (2001).

[16] UKQCD Collaboration, K.C. Bowler et al., Phys. Lett. B 486, 111 (2000).

[17] A. Abada et al., Nucl. Phys. B619, 565 (2001).

[18] JLQCD Collaboration, S. Aoki et al., Phys. Rev. D 64, 114505 (2001). 
[19] UKQCD Collaboration, D.R. Burford et al., Nucl. Phys. B447, 425 (1995).

[20] D. Becirevic, hep-ph/0211340.

[21] J. Shigemitsu et al., Phys. Rev. D 66, 074506 (2002).

[22] S.M. Ryan, Nucl. Phys. B (Proc. Suppl.) 106, 86 (2002).

[23] TrinLat Collaboration, G. Burgio, A. Feo, M.J. Peardon, and S.M. Ryan, Nucl. Phys. B (Proc. Suppl.) 119, 458 (2003).

[24] B. Alles, M. Campostrini, A. Feo, and H. Panagopoulos, Nucl. Phys. B413, 553 (1994).

[25] G. Burgio, S. Caracciolo, and A. Pelissetto, Nucl. Phys. B478, 687 (1996).

[26] F. Karsch, Nucl. Phys. B205, 285 (1982).

[27] M. Garcia Perez and P. van Baal, Phys. Lett. B 392, 163 (1997).

[28] TrinLat Collaboration, G. Burgio, A. Feo, M. J. Peardon, and S. M. Ryan (in preparation).

[29] H. J. Rothe, Lattice Gauge Theories, an Introduction, 2nd ed. (World Scientific, Singapore, 1997).

[30] K. Symanzik, Nucl. Phys. B226, 187 (1983).

[31] K. Symanzik, Nucl. Phys. B226, 205 (1983).

[32] M. Luscher and P. Weisz, Nucl. Phys. B452, 234 (1995).

[33] B. Alles, A. Feo, and H. Panagopoulos, Nucl. Phys. B491, 498 (1997).

[34] B. Alles, A. Feo, and H. Panagopoulos, Phys. Lett. B 426, 361 (1998).
[35] C. Christou, A. Feo, H. Panagopoulos, and E. Vicari, Nucl. Phys. B525, 387 (1998).

[36] E. Follana and H. Panagopoulos, Phys. Rev. D 63, 017501 (2001).

[37] R.K. Ellis and G. Martinelli, Nucl. Phys. B235, 93 (1984).

[38] M. Luscher and P. Weisz, Nucl. Phys. B445, 429 (1995).

[39] S. Caracciolo, A. Pelissetto, and A. Rago, Phys. Rev. D 64, 094506 (2001).

[40] G.P. Lepage and P.B. Mackenzie, Phys. Rev. D 48, 2250 (1993).

[41] H. Kawai, R. Nakayama, and K. Seo, Nucl. Phys. B189, 40 (1981).

[42] J.C. Collins, Renormalization (Cambridge University Press, Cambridge, England, 1984).

[43] T. Reisz, Commun. Math. Phys. 116, 573 (1988).

[44] T. Reisz, Commun. Math. Phys. 116, 81 (1988).

[45] S. Caracciolo, P. Menotti, and A. Pelissetto, Nucl. Phys. B375, 195 (1992).

[46] I.T. Drummond, A. Hart, R.R. Horgan, and L.C. Storoni, Phys. Rev. D 66, 094509 (2002).

[47] I.S. Gradshteyn and I.M. Ryzhik, Tables of Integrals, Series and Products, sixth ed. (Academic, San Diego, 2000).

[48] Handbook of Mathematical Functions, edited by M. Abramovitz and I.A. Stegun (Dover, New York, 1965). 